\section{Histoire Épistémologie Langage}

$42-2 \mid 2020$

Genèse, origine, récapitulation. Trân Đức Thảo face aux sciences du langage

\title{
Langues vivantes / langues mortes. Un paradigme en émergence au XVIIe siècle
}

\section{Gilles Siouffi}

\section{OpenEdition}

\section{Journals}

Édition électronique

URL : https://journals.openedition.org/hel/414

DOI : $10.4000 /$ hel. 414

ISSN : 1638-1580

Éditeur

Société d'histoire et d'épistémologie des sciences du langage

Édition imprimée

Date de publication : 31 décembre 2020

Pagination : 127-144

ISBN : 979-10-91587-13-6

ISSN : 0750-8069

Référence électronique

Gilles Siouffi, "Langues vivantes / langues mortes. Un paradigme en émergence au XVIle siècle », Histoire Épistémologie Langage [En ligne], 42-2 | 2020, mis en ligne le 28 octobre 2021, consulté le 09 janvier 2023. URL : http://journals.openedition.org/hel/414 ; DOI : https://doi.org/10.4000/hel.414

Ce document a été généré automatiquement le 9 janvier 2023.

Creative Commons - Attribution - Pas d'Utilisation Commerciale - Pas de Modification 4.0 International - CC BY-NC-ND 4.0

https://creativecommons.org/licenses/by-nc-nd/4.0/ 


\title{
Langues vivantes / langues mortes. Un paradigme en émergence au XVIIe siècle
}

\author{
Gilles Siouffi
}

1 La métaphore de la langue comme organisme vivant est classiquement citée comme une caractéristique typique $\mathrm{du} \mathrm{xIx}^{\mathrm{e}}$ siècle, et l'on mobilise généralement pour le montrer des citations emblématiques de Humboldt, Bopp et Schleicher, ce dernier ayant explicitement appliqué le paradigme darwinien au langage (Schleicher 1863). En France, l'enseignement des « langues vivantes » date de l'ordonnance de Vatimesnil de 1829 (Vatimesnil 1829). Depuis, on s'est habitué à séparer commodément les langues en deux ensembles, en rangeant dans les langues « mortes » le latin et le grec (ancien), et parfois d'autres langues, comme le sanskrit ou l'hébreu ancien, selon les contextes (scolaire ou scientifique), et dans les «langues vivantes» toutes les autres. Même si la terminologie de « langues anciennes » est la plus utilisée, la métaphore de la vie et de la mort est fréquemment mobilisée s'agissant des langues (Rey et Siouffi 2016). Il est significatif que Françoise Waquet ait introduit son enquête sur le devenir du latin (Waquet 1998) par une citation de Montherlant (Malatesta) évoquant la « langue morte » des personnages qu'il cherche à faire revivre. La métaphore a de fait rencontré une grande popularité dans la première moitié du $\mathrm{xx}^{\mathrm{e}}$ siècle. Pour autant, on se doute bien que le paradigme oppositionnel ne date pas du xixe siècle, ni les syntagmes eux-mêmes. Le nombre d'attestations relevées au XVII ${ }^{\mathrm{e}}$ siècle, en français, nous invite à mener une recherche plus approfondie sur cette période, même si les prémices peuvent en être recherchées encore plus haut.

De fait, le motif semble trouver sa source en Italie. Depuis l'opposition tracée par Dante entre ce qui est stable (le latin, appris par la grammaire) et ce qui est variable (les vernaculaires, appris par l'usage), ce premier paradigme s'est installé et déplacé. Selon Laurent Vallance, la première attestation, en italien, de l'expression lingua morta se trouve chez le grammairien italien Citolini en 1540: «che la [lingua]latina è morta, $e$ sepolta ne' libri ; e che la volgare è viva " (Lettera 6/33, cité dans Vallance $2019: 110)$. Peu de 
temps après, Sperone Speroni a également mobilisé l'adjectif (Speroni 1828 [1542] : 88) et Benedetto Varchi l'utilise pour classer les langues dans l'Ercolano (Varchi 1804 [1570] : 212-213; voir Faithfull 1953 et Yampolskaya 2016).

3 En France, les premières attestations significatives apparaissent dans la seconde moitié du XVI ${ }^{e}$ siècle. Depuis le De ratione studii d'Erasme (1512), le grec et le latin, auxquels on adjoindra bientôt l'hébreu, sont considérés comme des langues et comme des matières d'enseignement, ce qui donnera naissance aux trois collèges trilingues d'Alcalá, Louvain et Paris (voir Diu, 2018). Des langues fixées, certainement-mais ira-t-on jusqu'à les qualifier de "langues mortes"? On disait plutôt langues anciennes et modernes (notons que cette opposition, comme dans le titre du Trésor de Nicot s'appliquait aussi à l'opposition entre ce qui était perçu comme les deux états d'une même langue, comme le français).

4 La situation du latin et du grec est de fait singulière : ces langues ne sont certes plus en usage parlé, mais elles présentent tout de même des conditions de "vie ", pour ainsi dire. S'agissant des langues, la « mort » s'oppose-t-elle véritablement à la « vie »? C'est une question qui ne devra pas nous quitter au fil de notre parcours. Que signifie pour une langue être «morte » ou "vivante»? Les expressions sont-elles symétriques? Au $\mathrm{xVI}^{\mathrm{e}}$ siècle, le latin et le grec sont des langues « doctes", des langues de savoir qui ont à ce titre un certain usage, et elles sont aussi des langues liées à la transmission des Écritures. Le XVI ${ }^{\mathrm{e}}$ siècle paraît s'accommoder, dans une certaine mesure, de ce partage : vulgaires d'un côté, vraies « langues » de l'autre, sur lesquelles d'ailleurs, ou à partir desquelles, un travail grammatical est entrepris. Sont-elles pour autant « mortes»? Le diagnostic est difficile à établir, et si les paradigmes de la vie et de la mort sont employés, cela reste souvent dans un esprit métaphorique, dans un prolongement des nombreuses images tirées de la nature. «Le Maternel florit, dure, ou expire / Einsi que fét l'Etat, Réne, ou Ampire ", écrit par exemple Peletier du Mans dans sa Louange de la Parole (Peletier $1581: 12$ ).

5 C'est surtout au XVII siècle que le syntagme va s'installer, notamment après les réflexions de Vaugelas sur l'usage. La plupart des dictionnaires monolingues (Richelet 1680 ; Furetière 1690 ; Académie 1694) présentent les syntagmes langue vivante et langue morte au sein d'articles divers. Ces expressions, visiblement, se sont popularisées.

6 Nous proposons donc de mener l'enquête sur les usages de ces expressions, dans le domaine français, et sur leur mise en relation, aucune synthèse ne nous semblant avoir été menée à ce jour en dépit de recherches éparses (Colombat 1992a et 1992b notamment). Nous avons pour ce faire exploré systématiquement les corpus métalinguistiques réunis dans la base Garnier numérique ${ }^{1}$, ainsi que les textes numérisés dans Frantext ${ }^{2}$, en complétant avec d'autres citations. Cette méthode ne nous empêche pas d'être conscient des limites auxquelles peut se heurter ce type d'enquête. Il est toujours délicat, en effet, de mener une recherche historique sur l'usage d'un terme ou d'un syntagme: on peut se trouver piégé par des attestations visibles, les surévaluer, ou au contraire en manquer certaines d'essentielles.

7 En outre, quand on interroge ce qu'on pourrait appeler un «motif » - ici la vie et la mort des langues -, on remarque des retours de termes, on met en série des textes, mais est-on bien certain de repérer des évolutions, des articulations? On s'arrête sur des «moments ", mais est-on à même de saisir la continuité d'ensemble? Rien n'est moins sûr. 
8 Nous faisons néanmoins l'hypothèse d'une émergence de l'opposition langues vivantes / langues mortes entre le $\mathrm{xvI}^{\mathrm{e}}$ et le $\mathrm{xVII}^{\mathrm{e}}$ siècle. Nous allons donc essayer ici d'organiser un parcours qui, semble-t-il, révèle une évolution. Les enjeux de l'émergence de ce paradigme sont nombreux: il y va de la position des langues vernaculaires par rapport au latin, naturellement, mais aussi du rapport aux vicissitudes de l'usage dans sa relation aux « règles ", de la représentation des langues modernes, et même des langues en général, et de la transformation éventuelle des métaphores et images associées aux langues en une typologie.

9 Cette nouvelle confrontation des «langues vivantes » et des «langues mortes » fera ainsi apparaître que le XVII ${ }^{e}$ siècle se situe véritablement à la croisée des chemins dans son rapport aux langues.

\section{Le latin langue nouvellement morte?}

10 Comme l'indique Pascale Bourgain (2005: 38), le concept de langue morte n'existait pas au Moyen Age : on raisonnait plutôt sur celui de langue savante, avec lequel est venu graduellement contraster celui de langue vulgaire, dessinant l'opposition cardinale sur laquelle le $\mathrm{XvI}^{\mathrm{e}}$ siècle va fonder sa représentation des langues. Mais ce siècle est également hanté par le thème de la mutation, du changement, de la décadence, de la vicissitude. Les langues tombant dans la catégorie des choses humaines, elles sont soumises à la mue. On peut en lire un témoignage dans le chapitre connu de Louis Leroy sur la vicissitude des langues :

Semblablement les langues, parolles, escritures, caracteres, muent cõtinuellement, n'estans de meilleure condition que les autres choses humaines qui sont changees ordinairement avec leurs mots : à sçauoir manieres de viure publiques \& priuees, meurs, alimẽs, loix, habits, edifices, armes, machines, instrumens. Elles ont commencement, duree, perfection, corruption, alteration. Aucunes sont entierement perduës, les autres naissent des precedentes corrompues \& meslees. Les autres aprez auoir esté longuement aneanties sont restituees. Se maintiẽnent avec leur proprieté, elegãce, \& douceur : auec les sciences qui y sont escrittes, par la puissance \& grandeur d'Empire, par les religions: Auec lesquels moyens sont estendues en plusieurs païs, \& durent longuement : comme aussi elles se perdent par les contraires. (Leroy 1575, Livre I : $15 \mathrm{v}^{\circ}$ )

11 Autrement dit, il y a deux sorts possibles pour les langues : la mort, la perte (l'adjectif, ici, est perdues), et la « corruption », la dissolution dans autre chose. Ces mutations (les langues «muent continuellement») passent par différents stades qui peuvent être la perfection, la corruption ou altération, l'anéantissement (anéanties) ou la restitution, autrement dit, le fait que, quoique sorties d'usage, les langues échappent au néant grâce à l'usage qu'en font la science et la religion, et grâce à certaines conditions sociohistoriques particulières (« la puissance \& grandeur d'Empire »).

Le passage suivant glose dans son début les mêmes idées :

Si ne sont les lãgues de meilleure cõdition que les autres choses humaines: ains comme les edifices, habits, meurs, coustumes, loix, magistrats, manieres de viure publiques \& priuees, armes, machines, \& instrumens changẽt : ainsi font les mots, \& les langues qui perissent à la longue n'en demourant ny de leur escriture par succession de temps apparence quelconque. Nous n'auons aulcune cognoissance de la langue Osque, \& de l'Etrurienne. La Prouençale iadis celebree par nobles escriuains n'est plus entenduë des Prouençaulx du iourd'huy. L'ancienne Gauloise, Espagnole, Persienne, \& Punique perduës. (ibid. : $21 \mathrm{r}^{\circ}$ ). 

peut entendre par " mort o: faut-il comprendre la rupture dans la connaissance tout court (« nous n'avons aucune cognoissance »), ou la rupture dans la compréhension?
On retrouvera des propos similaires quelque temps plus tard chez Claude Duret : corrompus, icelle langue pareillement se changea \& corrompit, perdant sa nayfueté \& eloquence precedente soubs les Empereurs, puis estant l'Empire translaté de Rome à Constantinople, plusieurs nations estrangeres arriuants en Italie, altererent icelle langue tant qu'on laissa à la parler, \& est demeurée ez liures seulement, qui n'ont esté leuz n'y entendus plus de huict cents ans : les vns perdus, les autres mangez de vermolure, \& gastez de vieillesse, iusques à ce qu'aucuns Italiens \& Grecs ont par estude faict reuiure ces deux anciennes langues, assauoir la Latine \& Grecque, presque mortes ou perdues, descriuants, publiants \& corrigeants les liures restez ez bibliotheques garantie [sic] de la fureur \& rage des Barbares. (Duret 1613 : 803).

15 Si l'on revoit paraitre le motif de la corruption ou altération, on remarque que les deux adjectifs mortes et perdues sont associés, avec une modalisation significative (presque) qui exprime peut-être une réticence à franchir le pas. Pour Duret, le latin et le grec se signalent par une condition particulière: ils ont été «morts", puis on les a «fait revivre ». C'est le motif de la restitution des langues, associable à celui, plus général, de la « Renaissance ", terme encore absent à l'époque, mais auquel on confiera plus tard (à partir de 1828, selon le Dictionnaire historique de la langue française) la mission de caractériser l'époque où les savants européens ont prétendu parler et écrire latin et, dans une moindre mesure, grec, comme ces langues se parlaient et s'écrivaient à Rome ou en Grèce, et non plus comme les représentaient les clercs du Moyen Âge (voir Raffarin 2015).

De Ronsard, on peut également mobiliser un passage de la préface posthume de la Franciade (1587) :

C'est autre chose d'escrire en vne langue florissante qui est pour le present receuë du peuple, villes, bourgades \& citez, comme viue \& naturelle, approuuée des Rois, des Princes, des Senateurs, marchands \& trafiqueurs, \& de composer en vne langue morte, muette \& enseuelie sous le silence de tant d'espaces d'ans, laquelle ne s'apprend plus qu'à l'escole par le foüet \& par la lecture des liures, ausquelles langues mortes il n'est licite de rien innouer, disgraciées du temps, sans appuy d'Empereurs, ny de Rois, de Magistrats ny de villes, comme chose morte, laquelle s'est perduë par le fil des ans, ainsi que font toutes choses humaines, qui perissent vieilles, pour faire place aux autres suiuantes \& nouuelles. (Ronsard $1623: 588$ ).

Un adjectif apparaît ici comme équivalent possible à vivante: florissante, qui nous rappelle la vogue de cette image horticole à l'époque (bien que la forme de l'adjectif verbal florissant soit en voie de spécialisation dans le registre abstrait, se séparant ainsi de la forme fleurissant). Une langue florissante indique certainement un degré supérieur de vie.

Quant au syntagme langue morte (d'abord au singulier, puis au pluriel), il s'agit apparemment, à notre connaissance, de sa première attestation en français. La description est chargée, puisqu'on remarque également les mots muette, ensevelie, evanouie, perduë, le verbe périr... Non content d'avoir inscrit le motif, Ronsard le développe d'ailleurs et en propose une véritable analyse :

Le mesme Seigneur [le Turc] occupant par armes la meilleure partie de toute l'Europe, où on souloit parler la langue Latine, l'a totalement abolie, reduisant la 
Chrestienté, de si vaste \& grande qu'elle estoit, au petit pied, ne luy laissant presque que le nom, comme celle qui n'a plus que cinq ou six nations, où la langue Romaine se debite : \& n'eust esté le chant de nos Eglises, \& Psalmes, chantez au leuthrin, long temps y a que la langue Romaine se fust esuanouye, comme toutes choses humaines ont leurs cours : \& pour le iourd'huy vaut autant parler vn bon gros Latin, pourueu que l'on soit entendu, qu'vn affetté langage de Ciceron. Car on ne harangue plus deuant Empereurs, ne Senateurs Romains ; \& la langue Latine ne sert plus de rien, que pour nous truchementer en Allemaigne, Pologne, Angleterre, \& autres lieux de ce pays là. D'vne langue morte l'autre prend vie, ainsi qu'il plaist à l'arrest du Destin, \& à Dieu qui commande, lequel ne veut souffrir que les choses mortelles soient eternelles comme luy [...]. (ibid. : 589).

Pour Ronsard comme pour ses contemporains, la langue appartient à l'ensemble des « choses mortelles ». Mais cela ne signifie pas pour autant qu'on doive prendre la mort littéralement et totalement. Ronsard atteste ainsi la permanence d'un latin véhiculaire, signalant les limites de la « restauratio » humaniste de la langue de Cicéron : la langue se transforme, donne lieu à une autre vie. Il insiste également beaucoup sur le rapport entre la condition de la langue et le pouvoir politique.

Le latin peut-il donc être enregistré comme véritablement «mort»? Tout est affaire d'appréciation de la place désormais occupée, dans l'espace intellectuel et culturel, par une langue qui a été «perdue », puis « restituée ». S'agit-il du même «latin »? Il est clair ici, que les commentateurs se heurtent à la difficile mise en œuvre de l'individuation et de la démarcation des langues (Kremnitz 2008). Cette démarche liée au phénomène de la représentation rencontre des problèmes dans la diatopie (la différenciation des variétés et la différenciation entre langues et variétés), mais aussi dans la diachronie. Par latin, ne doit-on entendre que le latin ancien, le latin des Romains?

21 Au début du xvII siècle, Colombat (1992a ; Colombat \& Furno 1998) signale un épisode intéressant: la vive réaction que Jean Belot manifesta en 1637 à un passage de la préface des Nouvelles Conjectures sur la digestion de Marin Cureau de la Chambre dans laquelle l'auteur déploie, dans un esprit proche de celui de Descartes, en somme, un vibrant plaidoyer pour la langue française appliquée aux sciences, et laisse échapper, à propos du latin, la fatale épithète de morte:

Certainement à la voir Estrangere \& vagabonde, comme elle est par tout, à la voir toute morte qu'elle est, vsurper l'empire des Sciences \& des Lettres; ie me suis souuent imaginé que ce deuoit estre l'Ombre \& le Phantôme de ces vieux Tyrãs, qui sortoit de leurs tombeaux pour triompher de la liberté de nos Paroles \& de nos Pensées. (Cureau de La Chambre 1636 : xiii)

Cureau de La Chambre choisit explicitement d'exclure le latin des sciences et de la description de la nature, considérant que, dans ces matières, il faut imiter l'exemple de l'Antiquité et utiliser " sa langue naturelle », et estime que le latin n'est plus bon qu'à la Fable ou à l'Histoire :

I'aduouë que s'il me falloit écrire des Fables, ou faire l'Histoire du tẽps paßé, il me seroit à pardonner si j'employois des langues mortes, pour dire des choses qui ne sont plus, ou qui n'ont jamais esté. (ibid. : $\mathrm{x}$ )

Autrement dit, l'usage de la langue morte verrait son périmètre circonscrit aux choses du passé. Pour le présent, il faudrait user d'une langue vivante. Par ailleurs, on retrouve fréquemment dans son texte les métaphores de la jeunesse et de la vieillesse appliquées aux langues comme aux sciences, et de façon attendue, on revoit paraître le motif bien 
connu des langues assimilées aux plantes pour justifier la place accordée aux nouvelles venues :

Je sçay bien qu'il en est comme des Plantes Estrangeres, que l'on a toûjours grand peine d'eleuer, les vnes meurent, les autres languissent, mais außi celles qui peuuent souffrir ce changement, portent dans leur nouueauté des graces qui ne se trouuent point en toutes les autres. (ibid. : xvii)

L'Apologie de Belot, parue immédiatement après, se signale explicitement comme une "contestation» du texte de Cureau de La Chambre, selon le sous-titre et le mot de l'épître. On a donc affaire à une "mini-querelle ", annonçant, sur les mérites comparés du latin et du français, la future « querelle des inscriptions » de 1676 (voir Siouffi 2010). Dès la première page, Belot soutient que «les Langues qui ont été renduës les plus parfaites pour l'intelligence des hommes » ne peuvent connaître, à l'instar des empires, la disgrâce de tomber « dans la poussiere, \& dans le neant » (Belot 1637 : 2). Il reprend ensuite un à un tous les arguments évoqués par Cureau de La Chambre dans sa longue préface en réservant un commentaire particulier à l'adjectif morte qu'il a rencontré : "Cette belle Langue Latine n'est donc point morte comme il nous le veut persuader, \& s'il crêt l'auoir terrassée par le raisonement de sa Preface, qu'il ne doute pas de la voir comme vn Anthée reprendre de nouvelles forces... ». (ibid. : 35-36). Chez Cureau de La Chambre, le motif était présent de façon insistante, puisque le verbe périr, de son côté, se rencontrait dès la page 3 de la «Préface ». De fait, c'est bien l'aspect diagnostic du propos de Cureau de La Chambre qui choque Belot. Pour lui, le latin est immortel; il a des "graces qui ne flétriront jamais» (ibid.: 34); «sa viellesse ne connoit point de tâches, ny de rides » (ibid.); et puisque Cureau de La Chambre faisait partie de la toute nouvelle Académie, Belot clôt son court pamphlet par une lettre à ces «Messievrs ", à qui il prête des intentions peu claires sur ce sujet et qu'il presse d'épouser sa cause. Le latin nouvelle « langue morte»? Certains ne le voudraient certes pas.

\section{L'usage, maître et tyran des langues vivantes}

Si les premières manifestations de l'opposition langue vivante/langue morte au $\mathrm{xVI}^{\mathrm{e}}$ siècle ont pour objet principal le latin et son changement progressif de statut, la problématique va être enrichie au cours du XvII ${ }^{\mathrm{e}}$ siècle par le développement simultané des propositions de grammaires des langues modernes et des théories de l'usage.

Le problème essentiel qui va se présenter pour la grammaire, en effet, va désormais être de savoir comment dessiner les lignes d'une description stable et organisée s'agissant de langues soumises au changement. Pendant tout le Moyen Âge et jusqu'au $\mathrm{XVI}^{\mathrm{e}}$ siècle, il s'est construit une équivalence entre le latin langue savante et la démarche de la grammatica. Conçue sur des modèles scholastiques, la "grammaire » est censée mettre les utilisateurs de la langue à l'abri de sa variabilité. Se posant comme une discipline intellectuelle tendue dans une recherche rationnelle de règles, son travail sur les langues s'est largement fait à partir de ce statut d'étalon du latin, ce qui a donné lieu à une grammaire latine étendue, selon le terme d'Auroux (1992: 19), et a eu des répercussions autant sur la description des langues modernes que, singulièrement, sur celle du latin. «La grammaire latine occupe une place singulière dans les descriptions des langues naturelles. Place singulière qu'elle doit en particulier à son antériorité sur les descriptions des langues vivantes et à la progression du latin vers son statut actuel de langue morte", écrit Bernard Colombat (Colombat 1999: 9). Pour lui, les deux 
phénomènes sont connexes : disparition progressive du latin des usages vivants à partir de la fin du XVI ${ }^{\text {e }}$ siècle (il cite la Minerva de Sanctius parue en 1587 comme indice) et émergence d'une instrumentalisation de la grammaire latine aux fins de description des langues vernaculaires. Le latin est pour les humanistes une langue vivante, mais cela n'empêche pas qu'ils aient travaillé, objectivement, avec une langue "morte " (Tavoni $1984: 159$ et 160, cité par Colombat \& Furno 1998).

Mais graduellement, cette identification va être bousculée par le primat accordé à l'usage dans l'examen des langues modernes. La montée des théories de l'usage depuis le $\mathrm{xVI}^{\mathrm{e}}$ siècle a fait l'objet de nombreuses études (voir notamment Trudeau 1992). Sans doute la création de l'Académie a-t-elle été un tournant dans la mesure où l'article XXIV de ses statuts demandait à la Compagnie de travailler à "donner des règles certaines à notre langue " (Pellisson et D'Olivet 1858: vii). De fait, il semble que la cristallisation de la distinction langues vivantes / langues mortes date des années 1640, du moins dans les commentaires explicites que nous conservons.

Lorsqu'il fait paraître ses Remarques sur la langue françoise en 1647, en effet, Vaugelas, qui en avait déjà élaboré certaines dès les années 1610, met bien en avant qu'il s'agit pour lui de formuler des avis sur une langue vivante et non pas morte. Dès le début de la préface, il met en place sa définition décisive de «l'Usage, que chacun reconnoist pour le Maistre \& le Souverain des langues vivantes" (Vaugelas 1647, Préface, I), en une formule qu'il prendra le soin de rappeler dès la remarque qu'il fait figurer en tête de son recueil (ibid.:1), ce qui témoigne de l'importance qu'il accordait à ce précepte et de l'importance qu'il y avait selon lui à en pénétrer le lecteur.

Pour Vaugelas, deux ordres de phénomènes signalent la spécificité des langues vivantes par rapport aux langues mortes: la prééminence de l'oral et le caractère incontournable du changement. " la bonne prononciation [...] est une partie essentielle des langues vivantes ", écrit-il (ibid., Préface, II, 5) ; et « c'est la destinée de toutes les langues vivantes, d'estre sujettes au changement » (ibid., Préface, X).

Pour certains de ses commentateurs, cette prééminence de l'oral et cette importance du changement présentent un lien manifeste. Voici ce qu'écrit Louis Alemand, qui éditera après la mort de Vaugelas certaines de ses remarques restées inédites :

...les langues mortes ne sont pas sujettes, ou du moins elles le sont tres-peu à ces desordres que cause la difference qu'il y a entre l'écriture \& la prononciation. L'Hebraïque \& toute celles qui en ont esté formées, reçoivent la force \& la prononciation de leurs mots, des points qu'on place d'une differente maniere, suivant qu'on veut varier la signification, \& la prononciation des mots. La Grecque se servoit d'accens \& prononçoit quelques-unes de ses lettres differemment suivant les contrées ou Provinces differentes où elle étoit en usage. La Latine, étoit ce me semble, la moins sujette à toutes ces variations, \& il y a apparence que du tems d'Auguste on prononçoit justement les mots comme on les écrivoit, \& si on y trouve aujourd'huy quelque difference, elle vient absolument de la diversité des Nations qui se servent de cette maîtresse langue, \& qui l'accommodent au genie \& à la prononciation de la leur. (Alemand $1688: 12$ ).

31 Une typologie se construit, dont témoigne la préface du Tresor de recherches et antiqvitez gavloises et françoises de Pierre Borel (1655):

[Les langues] sont diuisées en viues \& en mortes, les mortes sont l'Hebraïque, la Grecque \& la Latine, qui n'estans plus que dans les Liures, \& estans à couuert du caprice des hommes qui les changent, ne sont plus sujettes à changement : mais les Langues viues sont celles qui sont en vsage pour le commerce des Nations, \& dont on se sert à présent dans chaque Royaume du Monde ; \& celles-cy peuuent estre 
appellées la corruption des Langues mortes dont elles ont tiré leur origine.

Or il n'y a point de Langue viue qui dans vn train ordinaire ne soit sujette au changement, quand bien il n'y en auroit aucune occasion Estrangère ; car la seule fantaisie des hommes qui s'ennuyent des vieux mots (comme de toutes les vieilles choses) est assez capable de les changer: Ce que le docte M. Menage a fort bien remarqué en sa requeste des Dictionnaires. (Borel $1655: 3$ )

La «tyrannie de l'usage » devient un principe accepté :

Or c'est vne maxime, que ceux qui trauaillent sur vne langue viuante, doiuent tousiours auoir deuant les yeux; Que les façons de parler qui sont authorisées par vn vsage general \& non contesté, doiuent passer pour bonnes... (Arnauld \& Lancelot $1660: 82-83)$

Pour Vaugelas, cette "tyrannie » explique que, même dans les langues «mortes », on trouve des irrégularités :

Surquoy il est à remarquer, que toutes les façons de parler, que l'vsage a establies contre les reigles de la Grammaire, tant s'en faut qu'elles soient vicieuses, ni qu'il les faille euiter, qu'au contraire on en doit estre curieux comme d'vn ornement de langage, qui se trouue en toutes les plus belles langues, mortes \& viuantes. (Vaugelas $1647: 305 / 265$ ).

Cette remarque, pour autant, ne fera pas l'unanimité et suscitera des objections de la part de Scipion Dupleix, Thomas Corneille et bien d'autres. Pour La Bruyère, qui s'interroge sur la valeur générale de «l'usage» dans les conduites humaines, la question se pose s'il faut vraiment assimiler la condition des langues modernes à « l'usage », ce qui reviendrait à démissionner devant les possibilités de la raison :

Est-ce donc faire pour le progrès d'une langue que de déférer à l'usage ? serait-il mieux de secouer le joug de son empire si despotique? faudrait-il dans une langue vivante écouter la seule raison qui prévient les équivoques, suit la racine des mots, et le rapport qu'ils ont avec les langues originaires dont ils sont sortis, si la raison d'ailleurs veut qu'on suive l'usage ? (La Bruyère $1696: 554$ )

La nouvelle importance accordée à l'usage vient par ailleurs perturber la représentation qu'on avait des langues anciennes. Celles-ci étaient-elles si "fixées » que cela? Les auteurs de la Nouvelle Méthode latine, par exemple, considèrent que « La plus exacte pureté de la langue Latine " était observable "lorsqu'elle estoit encore vivante » ([Lancelot] $1667: 456)$. Ménage estime qu'il est « quelquefois permis de faire des mots nouveaux dans les langues mortes » (lettre à Daniel Huet du 18 août 1663, cité dans Leroy-Turcan \& Wooldridge 1995 : 182).

D'un côté, le latin apparaît décidément comme une langue « morte », de l'autre, on doit lui reconnaître une certaine « vie », mais qui n'est plus la même. Le diagnostic est bien posé par Charles Sorel: "Ainsi la Langue Latine est une Langue morte, qui paroist viuante chez plusieurs Nations, quoy qu'elle ne soit plus propre à aucune Nation particulierement » (Sorel 1667 [1664] : 3). Le latin a gagné de sa " mort » une autre vie, non nationale, pour ainsi dire. Mais pour certains, cette nouvelle «vie» ne s'accompagne pas des inconvénients liés aux «changements » auxquels sont soumises les langues modernes. Vie d'un côté, mais stabilité de l'autre, ce qui cumule finalement tous les avantages :

[La langue latine] n'a pas non plus une autre imperfection des Langues vulgaires, qui estant de leur nature sujettes au changement, ne peuvent pas consequemment nous servir d'une regle certaine \& déterminée pour tous les siécles. Si elle est encore vivante par l'étenduë de son usage, elle a les avantages des Langues mortes, étant fixe \& arrêstée par un usage constant \& déterminé ; \& si son universalité la 
rend utile par tout, son immutabilité fait qu'elle pourra toûjours servir (Besnier 1674:9) démarche de rédaction d'un dictionnaire monolingue d'une langue moderne par le fait que le français égalerait les langues mortes: "[la langue françoise] est aujourdhui l'amour des Nations les plus polies ; \& elle dispute de la beauté avec toutes les langues mortes.» On retrouve ici le motif de la compétition - en dignité, en stabilité, en beauté - entre langues modernes et langues mortes, qui a parcouru les $\mathrm{xvI}^{\mathrm{e}}$ et XVII ${ }^{e}$ siècles, et qu'est venu manifester à nouveau, par exemple, l'épisode récent de la "querelle des inscriptions» (voir Charpentier 1676). «[la langue française] a quelque chose de noble \& d'auguste, qui l'égale presque à la langue Latine, \& la releve infiniment au-dessus de l'italienne et de l'espagnole, les seules langues vivantes qui peuvent raisonnablement entrer en concurrence avec elle ", écrit à la même époque Bouhours (1671:28).

On parvient ainsi à résoudre une aporie fondamentale du raisonnement sur les langues : l'emploi d'une langue comme le latin peut être « universel » sans que le latin soit pour autant une langue " vivante ". À la fin du XVII siècle, cette élaboration sur une condition empirique singulière vient rencontrer les spéculations contemporaines sur les langages artificiels et sur la possibilité de concevoir des langues susceptibles de n'être pas soumises à l'histoire.

«vivante» et certaines caractéristiques linguistiques? Autrement dit, l'opposition morte / vivante peut-elle construire une typologie ? Certains aimeraient le penser, mais la chose n'est pas si facile à montrer. Organisée en questions / réponses, la Grammaire de Claude Mauger, maître de langues installé à Londres, propose le petit échange suivant :

Mais qu'appellez vous Langues vivantes?

But what do you call living Tongues.

Toutes les Langues de toutes les Nations, si vous en exceptez les Langues Hebraique,

Grecque, \& Latine, qui n'ont point d'Auxiliaires. (Mauger $1684: 61$ ) portugais. "Il est vray", ajoute le maître. N'y aurait-il donc pas de ligne de partage possible?

L'opposition langue vivante / langue morte semble dessiner une ligne de partage dans le rapport à l'usage, mais non pas une ligne de partage grammaticale.

\section{L'enregistrement de l'opposition}

A la fin $\mathrm{du} \mathrm{XVII}^{\mathrm{e}}$ siècle, on trouve dans trois dictionnaires monolingues mention de la distinction, laquelle donne parfois lieu à de petits développements.

Dans le Dictionnaire de Richelet, à l'article SOUVERAIN, une citation de Vaugelas illustre une autre mention de l'un des deux syntagmes : "L'usage est le souverain dans les langues vivantes. Vau. Rem. C'est à dire, l'usage décide souverainement du langage. »

Ce poids des théories de l'usage se lit également dans l'exemple que Furetière enregistre à l'article FIXER: "Il est impossible de fixer les langues vivantes, d'empescher qu'elles ne changent. » Pour certains, cette impossibilité de «fixer » la 
langue est contraire à son aspiration à la stabilité et à la "perfection" (au sens d'« achèvement d'un processus »). On retrouve d'ailleurs le motif à l'article LANGUE : «Les langues vivantes sont celles qu'on peut apprendre par la fréquentation des peuples qui la [sic] parlent. L'usage est le tyran des langues vivantes » et il est complété à l'article USAGE :

Les langues vivantes s'apprennent plustost par l'usage, que par l'estude. Vaugelas a montré la difference du bon \& du mauvais usage, comment il falloit juger du bel usage; que l'usage étoit le roy, le tyran, le maistre, l'arbitre souverain des langues; que l'usage l'emportoit sur la raison, sur les regles de la Grammaire.

Ces quelques phrases témoignent du problème que pose la notion nouvelle de langue vivante à la conception alors dominante de la grammaire: l'usage, même s'il est tempéré par la définition restrictive d'un bon usage, est un facteur de perturbation dans la confiance en la raison qui est malgré tout venue soutenir, en cette fin de $\mathrm{XVII}^{\mathrm{e}}$ siècle, la plupart des discours scientifiques. S'agissant des langues, on se demande finalement si «l'étude » et la " grammaire » ne voient pas leurs périmètres limités au domaine étroit des « langues mortes ». Moment transitoire, qui précède la recherche bientôt active des principes : «Après m'être publiquement déclaré contre ce qu'on appelle l'Usage, dans la Langue Françoise, c'est à moi de soutenir le Principe ", déclarera bientôt Grimarest (1712: 1). Évoquant la figure d'un grammairien ayant en projet de rédiger une grammaire du français, selon ce qui est attendu de l'Académie, Fénelon déclarera à l'inverse: "Cette grammaire ne pourroit pas fixer une langue vivante; mais elle diminueroit peut-estre les changements capricieux par lesquels la mode règne sur les termes comme sur les habits. » (Fénelon 1852 [1715] : 4).

De son côté, la qualification du latin comme «langue morte» ne fait plus de doute, comme on le lit à l'article LATIN du Dictionnaire françois :

Langue morte \& celebre qu'on parloit autrefois à Rome, qui est à present le fondement de la litterature, \& connuë de tous les Sçavants, La Langue Latine passe par tout. En toute l'Europe on trouve des gens qui parlent Latin. Le Latin est la clef des sciences.

Du côté du premier dictionnaire de l'Académie (1694), on relèvera la même interrogation que chez Richelet et Furetière quant à la condition qui est celle d'une «langue vivante»:

Le Dictionnaire de l'Académie [...] a esté commencé et achevé dans le siecle le plus florissant de la Langue Françoise [...]. On dira peut-estre qu'on ne peut jamais s'asseurer qu'une Langue vivante soit parvenuë à sa derniere perfection; Mais ce n'a pas esté le sentiment de Ciceron, qui aprés avoir fait de longues reflexions sur cette matiere, n'a pas fait difficulté d'avancer que de son temps la Langue Latine estoit arrivée à un degré d'excellence où l'on ne pouvoit rien adjouster. (Préface)

Est-il opportun de proposer un dictionnaire d'une « langue vivante "? Dans l'aspiration à la « perfection », on lit à la fois l'ambition d'un accès à la valeur, l'espoir de la sortie d'une condition historique et celui d'une stabilité permettant une description juste et fiable.

Ces propos sont complétés par ce qu'on lit à l'article LANGUE :

On appelle, Langue vivante, Une langue que tout un peuple parle : \& Langue morte et grammaticale, celle qu'un peuple a parlé, mais qui n'est plus en usage que dans les livres. La Langue Françoise, la Langue Espagnole, sont des langues vivantes. La Langue Latine, la Langue Grecque litterale, sont des langues mortes. 
grammaticale. L'idée de "grammaire " au sens de grammatica s'élabore bien, toujours, sur la base d'un état fixé, " parfait », retiré de l'usage. En 1835, ce passage sera modifié de la manière suivante :

Langue morte, Celle qu'un peuple a parlée, mais qui n'existe plus que dans les livres.

Et, par opposition, Langue vivante, Celle qu'un peuple parle actuellement. On dit

dans le même sens Langue ancienne, par opposition à Langue moderne.

Le terme métalinguistique opposition signale bien, alors, la volonté de fixer les contours d'un système de désignation. Remarquons également l'apparition du couple parallèle langue ancienne / langue moderne. De fait, l'expression langue moderne ne se trouve pas chez les grammairiens et les remarqueurs de l'époque classique (Corpus Garnier), même si l'adjectif moderne a pu ici ou là être appliqué à l'époque à langue pour contraster un état de langue avec un état plus ancien, ou encore, fréquemment, cette fois, à usage. Dans ce sens typologique, le TLF cite des attestations chez Madame de Staël en 1810 et Nodier en 1831, et il semble que l'expression se soit répandue au $\mathrm{XIX}^{\mathrm{e}}$ siècle. Aujourd'hui, si le syntagme langues anciennes reste très attesté, celui de langues modernes l'est beaucoup moins, l'opposition ayant perdu de sa symétrie et s'étant visiblement défaite. $\mathrm{Au}$ début $\mathrm{du} \mathrm{xx}^{\mathrm{e}}$ siècle, on trouve également une équivalence entre langue ancienne et langue classique, une recherche spécifique restant à conduire sur cette dernière expression.

\section{Conclusion}

À la fin $\mathrm{du} \mathrm{XVII}^{\mathrm{e}}$ siècle, en France, le paradigme oppositionnel langue(s) vivante(s) / langue(s) morte(s) semble donc bien installé.

Nous avons vu que le processus a commencé au Xvi siècle, au travers d'un motif décliné dans une assez grande variété de termes : changement, naissance, vieillesse, le verbe périr, les participes perdu, évanoui, les substantifs corruption, altération... Les adjectifs mortes et vivantes (ou plus souvent vives) s'insèrent d'abord dans cette série de termes, puis deviennent des motifs en eux-mêmes, motifs commodes puisqu'ils présentent une symétrie, puis on observe que le discours se fige et s'organise autour de ces termes au $\mathrm{XVII}^{\mathrm{e}}$ siècle, donnant naissance à une véritable typologie.

Nous avons vu également que la popularité de l'idée vaugelasienne de « l'usage maître et tyran des règles " semble avoir joué un grand rôle dans l'installation de l'opposition, apportant un critère démarcatif entre langues "grammaticales », qui s'apprennent " par étude », et langues reposant essentiellement sur « l'usage ». C'est ainsi, au total, que la fin $d u X I_{I I}{ }^{e}$ siècle se représente les langues, du moins si l'on en croit la vulgate qui est proposée dans les trois grands dictionnaires monolingues, assez concordants sur ce point. L'opposition langue vivante / langue morte vient alors se superposer à celle entre usage et règle. L'usage serait-il dès lors synonyme de vie ? Et la règle de mort? En tout cas, si, au $\mathrm{XVI}^{\mathrm{e}}$ siècle, le statut de langue morte a pu apparaître à certains comme plutôt positif, car il supposait une « règle certaine ", et que la langue ne soit pas sujette au changement, la problématique de l'usage a tracé ensuite une nouvelle ligne de partage.

55 À l'une de nos questions de départ, le grec et le latin (secondairement l'hébreu) sont-ils des langues «mortes" ?, le $\mathrm{XVII}^{\mathrm{e}}$ siècle ne répondra pas toujours, de fait, de manière 
tranchée. On constatera tout d'abord que le grec a survécu, « sous une forme qui garde bien des traits de son apparence ancienne " (Colombat \& Furno 1998: 240), mais différente - c'est ce qu'on appellera plus tard le "grec moderne ». C'est ce qu'écrit d'ailleurs la philologue Anne Dacier :

Par tout ce que je viens de dire, on voit que la Langue Grecque a esté florissante jusqu'au quinziéme siécle, de sorte qu'elle estoit encore une Langue vivante il n'y a que deux cens soixante ans. Depuis ce temps-là encore nous avons eu des Grecs naturels tres sçavants. (Dacier $1714: 248$ )

De son côté, l'hébreu est classé comme " mort » car il est assimilé à l'Ancien Testament, alors que la poésie hébraïque médiévale, la littérature hassidique et la prose séculière ont été ignorées (Yampolskaya $2016: 18$ ).

$\mathrm{Au}$ bout du compte, on se retrouve avec un problème particulier pour le latin. Dans un premier temps, le latin s'est maintenu dans une condition intermédiaire, pour ainsi dire, ne pouvant être renvoyé à sa seule " ancienneté », puis, insensiblement, il a glissé vers la catégorie « langue morte », comme en témoignent les dictionnaires français du XVII ${ }^{e}$ siècle, ou l'avertissement du dictionnaire latin- français de Boudot (1704). «Lorsque la langue latine etoit une langue vivante, ceux qui vouloient faire des vers en cette langue connoissoient déja par l'usage la quantité : c'est-à-dire, la longueur ou la brieveté des syllabes", écrit l'abbé Du Bos (1733: 317); «Enfin une pierre abatit la statue ; c'est la langue latine qui cessa d'être une langue vivante ", écrit Du Marsais à la même époque (1730 : 242).

Que signifie dès lors être une " langue morte »? Et cela signifie-t-il la même chose que de "cesser d'être une langue vivante »? Comme nous l'avons vu, la caractérisation progressive du latin comme "langue morte» s'est accomplie au moment où la compréhension de ce qu'on pouvait mettre derrière le mot "grammaire " évoluait. D'abord fortement dépendante du caractère "fixé » du latin, la conception de la grammatica a ensuite dû faire face à la montée des théories de «l'usage » s'agissant des langues «non fixées». Être une «langue morte» représentait donc un certain avantage, si « morte » signifiait «non soumise aux vicissitudes du temps et à l'éternelle volonté de changement des hommes ». De ce point de vue, comme l'analyse Bernard Colombat (1992b), une langue morte a un rôle méthodologique, puisqu'on peut se donner l'impression d'y appliquer des règles en toute rigueur. Au xvIII siècle, cependant, il relève que, si ce rôle est encore reconnu par certains (Diderot), il sera contesté par d'autres (D'Alembert). Peut-être peut-on faire l'hypothèse que l'utilisation de l'adjectif morte (plutôt que fixe, réglée, ou grammaticale...), de ce point de vue, a joué un rôle. Une langue morte, après tout, ne suscite pas de connotations bien positives et on se prête bien vite à penser que l'exercice qu'on peut faire sur elle est mort lui aussi.

"Cesser d'être vivante », de son côté, est clairement négatif, comme on le perçoit dans la citation de Du Marsais. Et c'est ici, sans doute, que le constat de " cessation de vie " n'est pas synonyme du constat de «mort ». « Cesser d'être vivant », pour le latin, c'est cesser de présenter les opérativités du langage - opérativités sémantiques, pragmatiques et sociales - sur lesquelles l'âge classique (xVII ${ }^{e}$ et $\mathrm{xVIII}^{\mathrm{e}}$ siècles) a construit de nouveaux discours. Il ne s'agit plus alors de voir le latin en tant que langue dotée de grammaire, mais en tant que langage, idiome de communication, vecteur de l'expression humaine. De ce point de vue, seules les «langues vivantes » vont demeurer anthropologiquement dotées de valeur. C'est sur elles que s'appuie l'essor d'un discours 
qu'on caractérisera plus tard de «linguistique», renvoyant la notion de «langue morte » au seul contexte scolaire.

\section{BIBLIOGRAPHIE}

\section{Sources primaires}

Académie française. 1694. Le Dictionnaire de l'Académie françoise dédié au Roy. Paris : Jean-Baptiste Coignard.

Alemand, Louis-Augustin. 1688. Nouvelles observations, ou Guerre civile des François, sur la langue. Paris : Coignard.

Arnauld, Antoine et Claude Lancelot. 1660. Grammaire generale et raisonnée. Paris : Pierre Le Petit. Belot, Jean. 1637. Apologie de la langue latine contre la preface de Monsieur de La Chambre, en son livre des Nouvelles Conjectures de la digestion. Paris : François Targa.

Besnier, Pierre. 1674. La Réunion des langues, ou l'Art de les apprendre toutes par une seule. Paris : S. Mabre-Cramoisy.

Borel, Pierre. 1655. Tresor de recherches et antiquitez gauloises et françoises. Paris : Augustin Courbé. Boudot, Jean. 1704. Dictionnarium universale latino-gallicum. Paris : Jean Boudot. Bouhours, Dominique. 1671. Les Entretiens d'Ariste et d'Eugène. Paris : S. Mabre-Cramoisy. Charpentier, François. 1676. Deffense de la langue françoise pour l'inscription de l'Arc de triomphe. Paris : Barbin.

Citolini, Alessandro. 1540. Lettera in difesa della lingua volgare. Venise : Marcolini da Forlì. Cureau de La Chambre, Marin. 1636. Nouvelles Conjectures sur la digestion. Paris : Rocolet. Dacier, Anne. 1714. Des causes de la corruption du goust. Paris : Humbert.

Du Bos, Jean-Baptiste. 1733. Réflexions critiques sur la poésie et la peinture. Paris : Pierre-Jean Mariette.

Dumarsais, César Chesneau. 1730. Des tropes ou des diférens sens dans lesquels on peut prendre un même mot dans une même langue. Paris : Veuve Jean-Batiste Broca.

Duret, Claude. 1613. Thresor de lhistoire des langues de cest univers. Cologny : Berjon, pour la Société caldorienne.

Erasme. 1512. De duplici copia rerum ac verborum commentarii duo. Paris : Josse Bade.

Fénelon, François Salignac de la Mothe. 1852 [1715]. Lettre sur les occupations de l'Académie. Éd. Eugène Despois. Paris : Dezobry et Magdeleine.

Furetière, Antoine. 1690. Dictionnaire universel. Rotterdam : Arnout et Reinier Leers. 
Grimarest, Jean Léonor Le Gallois de. 1712. Eclaircissemens sur les principes de la langue françoise. Paris : Delaulne.

La Bruyère, Jean de. 1696. Les caractères de Théophraste traduits du grec, avec Les Caractères ou les mœurs de ce siècle. Paris : Michallet.

Lancelot, Claude. 1667. Nouvelle methode pour apprendre facilement la langue latine. Paris : Le Petit. Leroy, Louis. 1575. De la vicissitude ou variete des choses en l'univers (...). Paris : Pierre l'Huillier.

Mauger, Claude. [2014] 1684. Grammaire françoise de Claude Mauger avec des augmentations / French Grammar with additions. Londres (Nlle éd. critique établie par Valérie Raby. Paris : Classiques Garnier).

Peletier du Mans, Jacques. 1581. Oeuvres poetiques intitules louanges. Paris : Coloumbel.

Richelet, César-Pierre. 1680. Dictionnaire françois. Genève : Widerhold.

Ronsard, Pierre de. 1623. Les Euvres de Pierre de Ronsard. Paris : Macé.

Schleicher, August. 1863. Die Darwinsche Theorie und die Sprachwissenschaft - offenes Sendschreiben an Herrn Dr. Ernst Haeckel. Weimar : H. Böhlau.

Sorel, Charles. 1667 [1664]. La Bibliotheque françoise. $2^{\mathrm{e}}$ éd. Paris : Compagnie des libraires du Palais.

Speroni, Sperone. 1828 [1542]. Dialogo delle lingue. Alcune prose scelte di Sperone Speroni. Éd. par Bartolommeo Gamba. Venise : Tipografia di Alvisopoli.

Varchi, Benedetto. 1804 [1570]. L'Ercolano : dialogo di messer Benedetto Varchi nel quale si ragiona delle lingue, ed in particolare della Toscana e della Fiorentina. vol. 1. Milan : Società tipografica de' classici italiani.

Vatimesnil, Henri Lefebvre de. 2000 [1829]. Ordonnance du roi concernant l'administration supérieure de l'instruction publique, les facultés de Droit, les facultés de Médecine, les écoles secondaires de Médecine, les collèges royaux et communaux, les institutions et pensions et les écoles primaires protestantes (extraits). Réimpr. dans L'histoire et la géographie dans l'enseignement secondaire. Textes officiels. Tome 1: 1795-1914, 126. Paris : Institut national de recherche pédagogique.

Vaugelas, Claude Favre de. 2018 [1647]. Remarques sur la langue françoise. Paris : Veuve Camusat (Nlle éd. critique établie par Wendy Ayres-Bennett. Paris : Classiques Garnier).

\section{Sources secondaires}

Auroux, Sylvain, éd. 1992. Histoire des idées linguistiques $2:$ Le développement de la grammaire occidentale. Liège : Mardaga.

Bourgain, Pascale. 2005. Réflexions médiévales sur les langues de savoir. Tous vos gens à latin. Le latin, langue savante, langue mondaine ( $\mathrm{XVI}^{e}$-XVII ${ }^{e}$ siècles) dir. par Emmanuel Bury, 23-46. Genève : Droz.

Colombat, Bernard. 1992a. La description du latin à l'époque de la montée des vernaculaires. Histoire des idées linguistiques, t. 2, dir. par Sylvain Auroux, 509-521. Liège : Mardaga.

Colombat, Bernard. 1992b. Les XVII ${ }^{\mathrm{e}}$ et XVIII ${ }^{\mathrm{e}}$ siècles français face à la pédagogie du latin. Vita Latina $126: 30-43$.

Colombat, Bernard. 1999. La grammaire latine en France à la Renaissance et à l'âge classique. Théories et pédagogies. Grenoble : Ellug. 
Colombat, Bernard. \& Martine Furno. 1998. La mort du latin. Recherches et travaux 54 (Curiosité historique et intérêts philologiques. Hommage à Serge Lancel) : 223-240.

Diu, Isabelle. 2018. Erasme, du langage aux langues : à l'origine de la fondation des collèges trilingues. Fonder les savoirs, fonder les pouvoirs, $X V^{e}$-XVII ${ }^{e}$ siècles dir. Dominique de Courcelles, 5-16. Paris : Publications de l'École nationale des Chartes.

Faithfull, R. Glynn. 1953. The Concept of Living Language in Cinquecento Vernacular Philology. Modern Language Review 48 : 278-292.

Kremnitz, Georg. 2008. Sur la délimitation et l'individuation des langues. Avec des exemples principalement pris dans le domaine roman. Estudis romanics XXX : 7-38.

Leroy-Turcan, Isabelle \& Terence R. Wooldridge, dir. 1995. Gilles Ménage (1613-1692) grammairien et lexicographe. Lyon : Université Jean-Moulin \& SIHELDA.

Pellisson, Paul \& Pierre Joseph Thoulier D’Olivet. 1858. Histoire de l'Académie française. Paris : Didier.

Raffarin, Anne, éd. 2015. Débats humanistes sur la langue parlée dans l'Antiquité, textes de Flavio Biondo, Leonardo Bruni, Poggio Bracciolini et Lorenzo Valla, édités, traduits, présentés, annotés et commentés par Anne Raffarin. Paris : Les Belles Lettres.

Rey, Alain, dir., 1992. Dictionnaire historique de la langue française. Paris : Le Robert.

Rey, Alain \& Gilles Siouffi. 2016. De la nécessité du latin et du grec. Paris : Flammarion.

Siouffi, Gilles. 2010. Le " génie de la langue française ». Études sur les structures imaginaires de la description à l'âge classique. Paris : Champion.

Tavoni, Mirko. 1984. Lingua, grammatica, volgare. Padoue : Antenore.

Trudeau, Danielle. 1992. Les inventeurs du bon usage, 1529-1647. Paris : Minuit.

Vallance, Laurent. 2019. Les grammairiens italiens face à leur langue (15e-16e s.). Berlin : De Gruyter.

Waquet, Françoise. 1998. Le Latin ou l'empire d'un signe, $\mathrm{XVI}^{e}-\mathrm{XX}{ }^{e}$ siècles. Paris : Albin Michel.

Yampolskaya, Sonya B. 2016. The Concept of "dead language" as exemplified by Hebrew. ВЕСТник СПБГУ. СЕР. 13. ВОСТОКОВЕДЕНИЕ. АФРИКАНИСТИКА $3: 16-30$.

\section{NOTES}

1. Cf. Grand Corpus des grammaires françaises, des remarques et des traités sur la langue (XIV $\left.{ }^{e}-X V I I^{e} s.\right)$,

B. Colombat, J.-M. Fournier, W. Ayres-Bennett (dir.), Classiques Garnier numérique (depuis 2011).

2. Frantext est une base de textes français de toute époque développée par le laboratoire Atilf (Analyse et traitement informatique de la langue française) à Nancy. 


\section{RÉSUMÉS}

L'article étudie l'émergence des qualifications langue morte et langue vivante en France au $\mathrm{XVII}^{\mathrm{e}}$ siècle. Il s'appuie sur un dépouillement de sources métalinguistiques (grammaires, dictionnaires, recueils de remarques, traités divers). Une première partie traite de l'évolution des termes pour qualifier le latin et montre comment on est passé du motif de l'altération et de la corruption, présent depuis le $\mathrm{xVI}^{\mathrm{e}}$ siècle, vers celui de langue morte. Une deuxième partie montre comment le développement des théories de l'usage est concomitant de la valorisation nouvelle des langues vivantes. Une troisième partie montre comment les dictionnaires de la fin du siècle enregistrent l'opposition langue vivante / langue morte, ouvrant la voie à une manière d'organiser la représentation des langues qui se diffusera dans le contexte scolaire. Au travers de l'étude de ce paradigme émergent, c'est la question de la représentation des langues comme dotées de grammaires réglées ou comme vecteurs changeants de l'expression humaine qui est abordée.

This paper examines the emergence of the qualifications langue morte (dead language) and langue vivante (living language) in France in the 17th century. It is based on an extensive study of metalinguistic sources (grammars, dictionaries, collections of remarks, various treatises). A first part deals with the evolution of the notions used to qualify Latin, from a representation in terms of alteration and corruption, in use since the 16th century, to those of life and death. The second part shows how the development of the theory of usage is concomitant with a new valorisation of living languages. The third part shows how dictionaries of the end of the century record the opposition between langue vivante and langue morte, paving the way for a new representation of languages that would later spread to educational contexts. Through the study of this emerging paradigm, the question of the representation of languages as having regulated grammars or as changing vectors of human expression is addressed.

\section{INDEX}

Mots-clés : langue vivante, langue morte, grammaire, idiome, usage

Keywords : living language, dead language, grammar, idiom, language use

\section{AUTEUR}

\section{GILLES SIOUFFI}

Sorbonne Université, Sens Texte Informatique Histoire (EA 4509, STIH), Paris, France 